\title{
STM-Induced Hydrogen Desorption via a Hole Resonance
}

Stokbro, Kurt; Thirstrup, C.; Sakurai, M.; Quaade, Ulrich; Hu, Ben Yu-Kuang; Perez-Murano, F.; Grey, Francois

Published in:

Physical Review Letters

Link to article, DOI:

10.1103/PhysRevLett.80.2618

Publication date:

1998

Document Version

Publisher's PDF, also known as Version of record

Link back to DTU Orbit

Citation (APA):

Stokbro, K., Thirstrup, C., Sakurai, M., Quaade, U., Hu, B. Y-K., Perez-Murano, F., \& Grey, F. (1998). STMInduced Hydrogen Desorption via a Hole Resonance. Physical Review Letters, 80(12), 2618-2621.

https://doi.org/10.1103/PhysRevLett.80.2618

\section{General rights}

Copyright and moral rights for the publications made accessible in the public portal are retained by the authors and/or other copyright owners and it is a condition of accessing publications that users recognise and abide by the legal requirements associated with these rights.

- Users may download and print one copy of any publication from the public portal for the purpose of private study or research.

- You may not further distribute the material or use it for any profit-making activity or commercial gain

- You may freely distribute the URL identifying the publication in the public portal

If you believe that this document breaches copyright please contact us providing details, and we will remove access to the work immediately and investigate your claim 


\title{
STM-Induced Hydrogen Desorption via a Hole Resonance
}

\author{
K. Stokbro, ${ }^{1}$ C. Thirstrup, ${ }^{2}$ M. Sakurai, ${ }^{2}$ U. Quaade, ${ }^{1}$ Ben Yu-Kuang Hu, ${ }^{1}$ F. Perez-Murano, ${ }^{1,3}$ and F. Grey ${ }^{1}$ \\ ${ }^{1}$ Mikroelektronik Centret, Danmarks Tekniske Universitet, Bygning 345 \\ ${ }^{2}$ Surface and Interface Laboratory, RIKEN, Saitama 351, Japan \\ ${ }^{3}$ Department of Electronic Engineering, Universitat Autonoma de Barcelona, 08193 Bellaterra, Spain
}

(Received 10 October 1997)

We report STM-induced desorption of $\mathrm{H}$ from $\mathrm{Si}(100)-\mathrm{H}(2 \times 1)$ at negative sample bias. The desorption rate exhibits a power-law dependence on current and a maximum desorption rate at $-7 \mathrm{~V}$. The desorption is explained by vibrational heating of $\mathrm{H}$ due to inelastic scattering of tunneling holes with the $\mathrm{Si}-\mathrm{H} 5 \sigma$ hole resonance. The dependence of desorption rate on current and bias is analyzed using a novel approach for calculating inelastic scattering, which includes the effect of the electric field between tip and sample. We show that the maximum desorption rate at $-7 \mathrm{~V}$ is due to a maximum fraction of inelastically scattered electrons at the onset of the field emission regime. [S00319007(98)05616-6]

PACS numbers: 61.16.Ch, 68.10.Jy, 79.20.La, 81.65.Cf

Single atom manipulation with a scanning tunneling microscope (STM) has been reported for several systems and a variety of physical mechanisms has been proposed to account for such manipulation [1,2]. Among the works relevant for the present Letter we mention single hydrogen atom desorption from the $\mathrm{Si}(100)-\mathrm{H}(2 \times 1)$ surface [3], and dissociation of single $\mathrm{O}_{2}$ molecules on $\mathrm{Pt}(111)$ [4]. These manipulations were performed at positive sample bias, and the underlying microscopic mechanism has been related to vibrational heating by inelastic scattering of tunneling electrons with an electron resonance on the sample. There have been theoretical predictions that a related mechanism may operate at negative sample bias [5], which involve inelastic scattering of a tunneling hole with a hole resonance on the sample. However, to our knowledge there has been no experimental confirmation of such a mechanism, probably because high tunnel currents and sample biases are needed to obtain high inelastic scattering rates with low-lying hole resonances (see Fig. 1).

In this Letter, we present evidence for a desorption mechanism involving a hole resonance, for STMinduced hydrogen desorption from the monohydride $\mathrm{Si}(100)-\mathrm{H}(2 \times 1)$ surface in ultrahigh vacuum [3]. Whereas in previous studies [3,6,7], hydrogen desorption has been studied at positive sample bias, here we report hydrogen desorption at negative bias. The desorption process is modeled by vibrational heating of hydrogen caused by inelastic scattering of tunneling holes with the $\mathrm{Si}-\mathrm{H} 5 \sigma$ hole resonance. The inelastic scattering rates are calculated using a novel method based on first principles electronic structure theory, and desorption rates obtained by solving the Pauli master equation for a truncated harmonic potential well [8]. With this model we obtain desorption rates as function of tunnel current and sample bias that are in agreement with the experimental data. We find a maximum desorption rate at sample bias $-7 \mathrm{~V}$, which coincides with the onset of the field emission or Fowler-Nordheim regime $[9,10]$. We show that this results from a competition between polarization of the hole resonance, which increases the fraction of inelastic scattered electrons, and domination of Fermi-level contributions to the tunnel current in the field emission regime.

The experiments were performed on $n$-type $\mathrm{Si}(100)$ $\left(N_{D}=1 \times 10^{18} \mathrm{~cm}^{-3}\right)$ samples using a JEOL JSTM-4000XV microscope at a base pressure of $1 \times 10^{-10}$ torr. Atomic hydrogen was absorbed on the clean $\mathrm{Si}(100)-(2 \times 1)$ reconstructed surface to obtain the monohydride $(2 \times 1)$ phase, in a manner identical to previous reports [7], and $\mathrm{W}$ tips were used. The desorption experiments were carried out by scanning the STM tip at speed, $s$, sample bias, $V_{b}$, and tunnel current, $I$, and subsequently imaging the affected area to determine the number of Si sites where desorption occurred. Figure 2 shows a typical example of desorption at negative sample bias of hydrogen along a single dimer row. We first
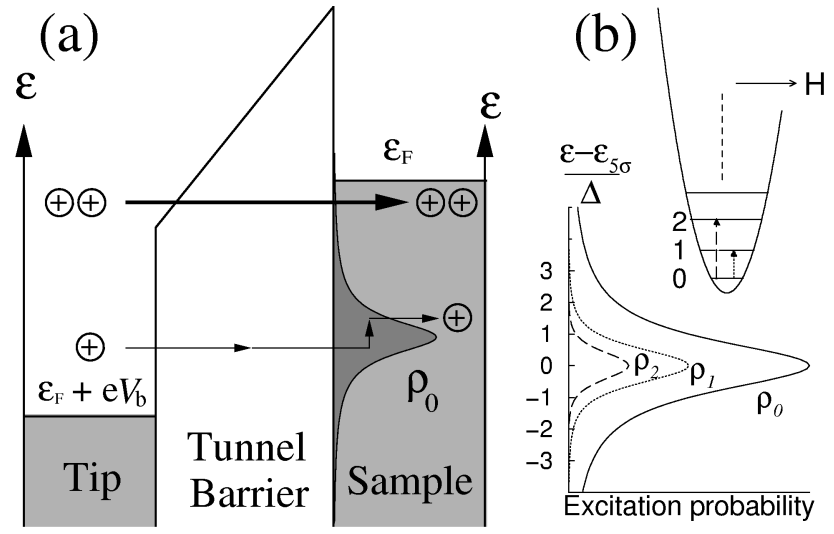

FIG. 1. (a) Inelastic tunneling of a hole into an adsorbate induced hole resonance with density of states, $\rho_{0}$. The higher barrier for tunneling into the hole resonance compared to tunneling into Fermi-level states, means that only a fraction of the total tunnel current will pass through the hole resonance. (b) Schematic illustration of relative energy dependent probabilities, $\rho_{n}(\varepsilon)$, for inelastic hole tunneling with energy transfer $n \hbar \omega_{0}$ to the adsorbate. 

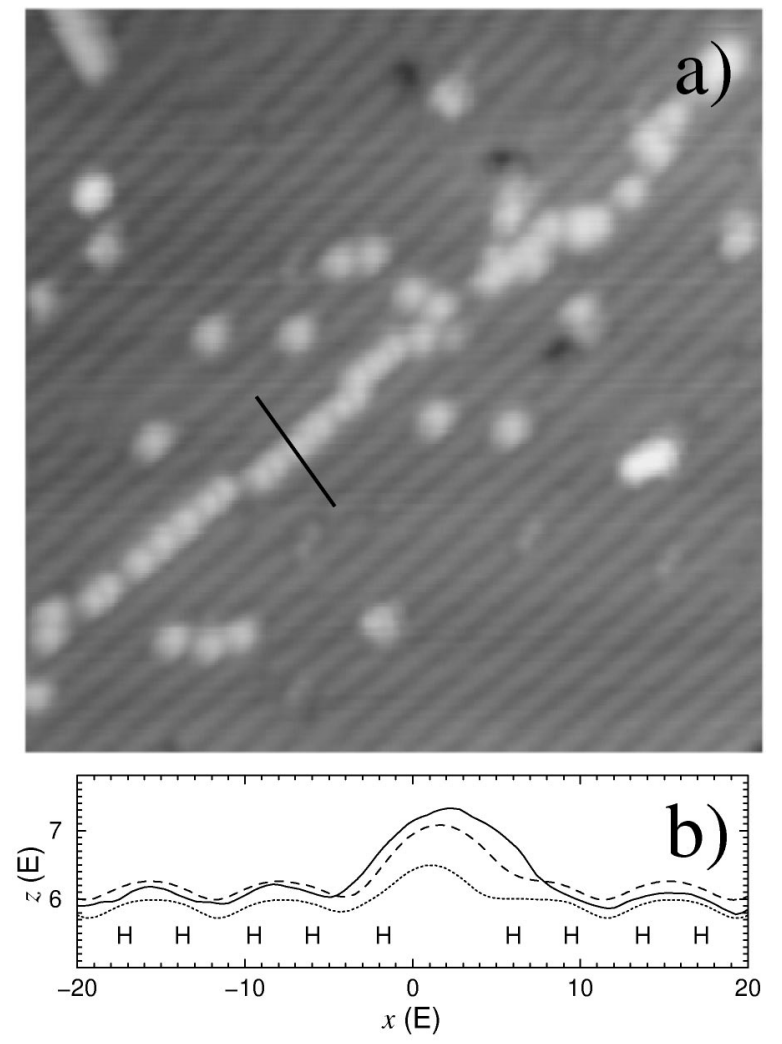

FIG. 2. (a) A single line of desorbed $H$ from the $\mathrm{Si}(100)-\mathrm{H}(2 \times 1)$ surface as a result of a line scan at $-7 \mathrm{~V}$ and $3.0 \mathrm{nA}$. The picture is obtained at $-2 \mathrm{~V}$ and $0.2 \mathrm{nA}$, and bright regions are due to increased density of states of $\mathrm{H}$-free Si atoms. (b) The experimental corrugation (solid line) along the line in (a) compared with the theoretical corrugation of a missing $\mathrm{H}$ defect including electric field effects (dashed line) and without electric field effects (dotted line). Results in the range $-7 \AA<x<9 \AA$ are calculated using a $c(4 \times 4)$ cell with one missing $\mathrm{H}$ atom and outside this range using a $(2 \times 1)$ cell.

measured the dependence of the desorption rate, $R$, on the tunnel current for sample biases of $V_{b}=-7$ and $-5 \mathrm{~V}$. The results are shown in Fig. 3 (inset). For both biases there is a power-law dependence of the desorption rate upon current, $R=R_{0}\left(I / I_{\mathrm{des}}\right)^{\alpha}$, with exponent $\alpha \approx 6$. In this equation $I_{\mathrm{des}}$ is the tunnel current that gives rise to a fixed desorption rate $R_{0}$. In order to find the voltage dependence of the desorption rate, we measure $I_{\text {des }}\left(V_{b}\right)$ with $V_{b}$ in the range -10 to $-4 \mathrm{~V}$, as shown in Fig. 3. The measurements were obtained by scanning $30 \mathrm{~nm}$ along a single dimer row at $s=2 \mathrm{~nm} / \mathrm{s}$, and $I_{\text {des }}$ is defined as the current that gives rise to desorption of $50 \%$ of the hydrogen along such a scan line, which corresponds to $R_{0}=4 \mathrm{~s}^{-1}$.

The main feature of Fig. 3 is that significantly higher bias and tunnel currents are required at negative bias, compared to the positive bias case [3]. Whereas $I_{\text {des }}\left(V_{b}\right)$ decreases monotonically at positive $V_{b}$ [3], it displays a minimum at negative $V_{b}$, increasing for $V_{b}<-7 \mathrm{~V}$. Experimentally, the lithographic resolution at negative bias is comparable to that at positive bias. At the highest

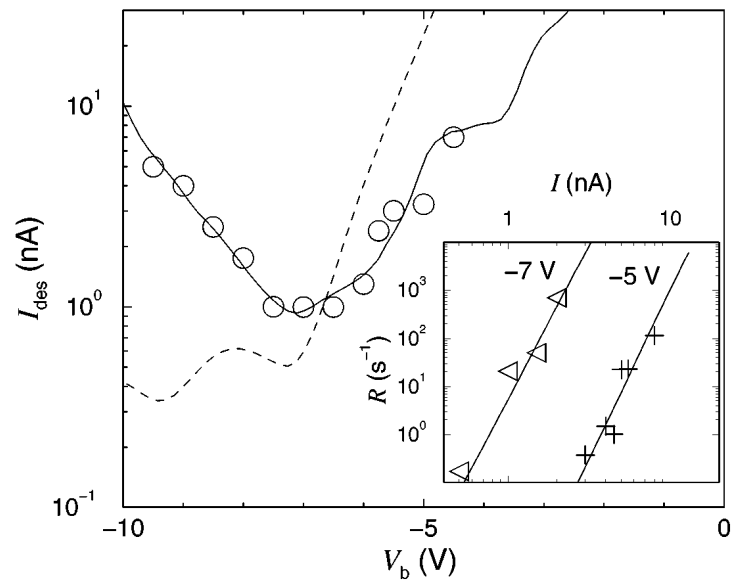

FIG. 3. Current, $I_{\text {des }}$, as a function of sample bias, $V_{b}$, for constant desorption rate $R=4 \mathrm{~s}^{-1}$. Circles show experimental results, and the solid line shows the theoretical result. The dashed line shows the current as a function of sample bias for constant electric field $E=0.8 \mathrm{~V} / \AA$ in the tunnel gap. Inset shows $R(I)$ for $V_{b}=-7 \mathrm{~V}$ (triangles) and $-5 \mathrm{~V}$ (crosses), and from least-squares fits of $R \propto I^{\alpha}$ to the data we obtain $\alpha=5.7 \pm 0.7(-7 \mathrm{~V})$ and $6.3 \pm 1.3(-5 \mathrm{~V})$. Lines show theoretical calculations and have exponents $\alpha=6.4(-7 \mathrm{~V})$ and $7.1(-5 \mathrm{~V})$.

current levels there is a tendency for the tip resolution to be affected by the lithography process. This limits the voltage range over which $I_{\text {des }}\left(V_{b}\right)$ can be readily measured, and may explain why no detailed investigation of desorption at negative sample bias has been made previously. We note that the behavior of $I_{\mathrm{des}}\left(V_{b}\right)$ at negative sample bias has been confirmed independently on a separate STM system [11].

At negative sample bias, electrons accelerated across the tunnel gap impinge on the tip, so the possibility of direct excitation by collision can be ruled out as a desorption mechanism. In contrast, at positive sample bias, direct excitation of the $\mathrm{Si}-\mathrm{H}$ bond is believed to play the dominant role for $V_{b}>4 \mathrm{~V}[3,6]$. Shen et al. [3] have proposed vibrational heating of hydrogen [12] by tunneling electrons scattering inelastically with the $\mathrm{Si}-\mathrm{H}$ $6 \sigma^{*}$ electron resonance as a desorption mechanism for positive sample bias and $V_{b}<4 \mathrm{~V}$. A similar mechanism can function at negative sample bias, since an electron tunneling from the sample to the tip may excite the $\mathrm{Si}-\mathrm{H}$ $5 \sigma$ hole resonance, which upon deexcitation can transfer energy to the hydrogen atom [5]. This process can be viewed as inelastic scattering of a tunneling hole with the Si-H $5 \sigma$ hole resonance, as illustrated in Fig. 1. A characteristic feature of vibrational heating by inelastic scattering is a power-law dependence of the desorption rate on current [13] in agreement with the experimental observations.

To make a quantitative analysis of the dependence of the desorption rate on tunnel current and sample bias, we develop a method for obtaining inelastic scattering rates from first principles electronic structure calculations. Since the inelastic scattering rate depends on the tip-sample 
distance, $d$, while the measured quantities are $I$ and $V_{b}$, we first calculate the relation between $I, V_{b}$, and $d$. For this purpose we use a high voltage extension of the work by Tersoff and Hamann [14], that includes the effect of the electric field between tip and sample [15]. The tunnel current is obtained from the local density of states (DOS) of the $\mathrm{Si}(100)-\mathrm{H}(2 \times 1)$ surface, $\rho(\mathbf{r}, \varepsilon, E)=\sum_{\mu}\left|\psi_{\mu}(\mathbf{r}, E)\right|^{2} \delta\left(\varepsilon-\varepsilon_{\mu}\right)$, at tip position $\mathbf{r}$, and wave functions $\psi_{\mu}$ for electrons with energy $\varepsilon_{\mu}$ are calculated in an external field $E$. The electric field is modeled by a planar electric field outside the surface and its magnitude determined from $V_{b}$ and $d$. The tunnel current is given by [15]

$$
I=C_{\mathrm{W}} \int_{0}^{e V_{b}}\left|e^{2 R_{\mathrm{w}} \kappa(\varepsilon)}\right| \rho\left(d+R_{\mathrm{w}}, \varepsilon, E\right) d \varepsilon,
$$

where distances are in bohrs, current in amperes, and all electron energies are relative to the sample Fermi energy, $\varepsilon_{\mathrm{F}}$. In this equation $\kappa(\varepsilon)=\sqrt{2 m\left(\phi_{t}+e V_{b}-\varepsilon\right)} / \hbar$ is the wave function inverse decay length, $\phi_{t}=4.5 \mathrm{eV}$ the work function of the W tip [16], $R_{\mathrm{W}}=3$ a.u. the atomic radius of $\mathrm{W}$, and the normalization constant $C_{\mathrm{W}}=$ $0.007 R_{\mathrm{W}}^{2}$ amperes $\times$ bohr is obtained from a calculation of a model W tip [15].

We test the method by calculating the STM corrugation across a single missing $\mathrm{H}$ defect. The electronic structure calculations are based on density-functional theory $[17,18]$ within the generalized-gradient approximation (GGA) [19] using 20 Ry plane-wave basis sets. The $\mathrm{Si}(100)-\mathrm{H}(2 \times 1)$ surface is represented by a 12 layer $(2 \times 1)$ slab, and we use a $c(4 \times 4)$ slab to calculate the corrugation of a single missing $\mathrm{H}$ defect. Ultrasoft pseudo-potentials [20] are used for both $\mathrm{H}$ and $\mathrm{Si}$. Figure 2(b) shows the result compared with the measured corrugation perpendicular to a desorption line. The theoretical corrugation compares well with the experimental data when the electric field between tip and sample is included (dashed line) but not otherwise (dotted line).

We next calculate the tunnel current of inelastically scattered electrons. Electrons may scatter inelastically due to long-range electrostatic interactions with a vibrational transition dipole moment, or due to local inelastic scattering with an electronic resonance [21]. Only a local interaction can cause atomic scale desorption, so in the following we restrict the analysis to resonance coupling. The resonance scattering event can be described by a local polaron model with a linear electron-phonon coupling $\lambda$, and we use a harmonic approximation for the $\mathrm{Si}-\mathrm{H}$ bond potential with frequency $\omega_{0}$. To calculate the inelastic current, $I_{n}$, which causes transitions from vibrational level 0 to $n$, we combine previous models of inelastic-scattering $[5,22]$ with the Tersoff-Hamann model of STM tunneling between a surface and a W tip $[14,15]$. In the limit of a broad resonance, $\Delta \gg \lambda, \omega_{0}$, we obtain [23]

$$
I_{n}=C_{\mathrm{W}} n ! \int_{n \hbar \omega_{0}}^{e V_{b}}\left|e^{2 R_{\mathrm{w}} \kappa(\varepsilon)}\right| \rho_{n}\left(\mathbf{d}+R_{\mathrm{w}}, \varepsilon, E\right) d \varepsilon,
$$

where distances are in a.u. and $I_{n}$ in amperes. We define a dimensionless parameter, $K=\pi \lambda^{2} \rho_{s} / \Delta$, where $\rho_{s}=$ $\sum_{\mu} \delta\left(\varepsilon_{5 \sigma}-\varepsilon_{\mu}\right)$ is the average surface DOS around the resonance energy $\varepsilon_{5 \sigma}$, and $\Delta$ is the resonance width. The weighted local DOS,

$\rho_{n}(\mathbf{r}, \varepsilon, E)=K^{n} \sum_{\mu} f_{\mu}|\langle 5 \sigma \mid \mu\rangle|^{2 n}\left|\psi_{\mu}(\mathbf{r}, E)\right|^{2} \delta\left(\varepsilon-\varepsilon_{\mu}\right)$,

is weighted with $\left(K\langle 5 \sigma \mid \mu\rangle^{2}\right)^{n}$, where $|5 \sigma\rangle$ and $|\mu\rangle$ are the resonance state and sample eigenstates, respectively, and $f_{\mu}=|\langle 5 \sigma \mid \mu\rangle|^{2} /\left(x+|\langle 5 \sigma \mid \mu\rangle|^{2}\right)$ is the fraction of electrons which tunnel from the tip to state $\mu$ via the $5 \sigma$ resonance. The parameter $x$ determines the fraction of electrons which does not tunnel via the resonance state, and we have estimated $x \approx 0.1(=0.25 \times$ $\left.\max _{\mu}|\langle 5 \sigma \mid \mu\rangle|^{2}\right)$. We note that the calculations are quite insensitive to the value of $x$, since $f_{\mu} \sim 1$ for $\varepsilon_{\mu} \sim$ $\varepsilon_{5 \sigma}$ and $f_{\mu} \sim|\langle 5 \sigma \mid \mu\rangle|^{2} / x$ for $\left|\varepsilon_{\mu}-\varepsilon_{5 \sigma}\right| \gg \Delta$, thus $x$ merely damps contributions from eigenstates not in resonance.

We next calculate the parameters entering Eq. (2) for inelastic scattering with the $\mathrm{Si}-\mathrm{H} 5 \sigma$ resonance. From a frozen phonon calculation we obtain $\hbar \omega_{0}=0.26 \mathrm{eV}$. Using the ground state energy of a free $\mathrm{H}$ atom we calculate $E_{\mathrm{des}}=3.36 \mathrm{eV}$. To find $\langle 5 \sigma \mid \mu\rangle$ we project the electronic eigenstates of the slab calculation onto the $5 \sigma$ wave function of a Si-H molecule, and the solid line in Fig. 4 shows the partial DOS $n_{5 \sigma}(\varepsilon)=\sum_{\mu}|\langle 5 \sigma \mid \mu\rangle|^{2} \delta(\varepsilon-$ $\left.\varepsilon_{\mu}\right)$. We find that the $5 \sigma$ resonance is centered at $\varepsilon_{5 \sigma}=$ $-5.3 \mathrm{eV}$ relative to the Fermi level of an $n$-type sample with an average width of $\Delta=0.6 \mathrm{eV}$. Crosses in Fig. 4 show $\rho_{s}\left(\varepsilon_{\mu}\right) \approx n\left(\varepsilon_{\mu}\right) /|\langle 5 \sigma \mid \mu\rangle|^{2}$ from which we estimate $\rho_{s} \approx 1.2 \mathrm{eV}^{-1}$. The electron-phonon coupling is given by $\lambda=\varepsilon_{5 \sigma}^{\prime} \sqrt{\hbar / 2 M \omega_{0}}$, where $\mathrm{M}$ is the hydrogen mass and $\varepsilon_{5 \sigma}^{\prime}$ the derivative of $\varepsilon_{5 \sigma}$ with respect to the $\mathrm{Si}-\mathrm{H}$ bond length $z$ [5]. Our calculations show that $\varepsilon_{5 \sigma}$ varies almost linearly with $z$ in the range $1.25 \AA<z<$ $2.25 \AA$ with a slope $\varepsilon_{5 \sigma}^{\prime}=2.3 \pm 0.1 \mathrm{eV} / \AA$, and hence $\lambda \approx 0.20 \mathrm{eV}$.

From the inelastic currents we calculate desorption rates by solving the Pauli master equation for the transitions among the various levels of the oscillator [8]. We include contributions from $I_{1}, I_{2}$, and $I_{3}$ [24], and vibrational relaxations are described by a current independent relaxation rate, $\gamma=1 \times 10^{8} \mathrm{~s}^{-1}[3,25]$. We assume that desorption occurs when the energy of the $\mathrm{H}$ atom exceeds the desorption energy $E_{\text {des }}=3.36 \mathrm{eV}$, corresponding to a truncated harmonic potential well with 13 levels. The solid lines in Fig. 3 (inset) show the calculated desorption rate as function of tunnel current for $V_{b}=-5$ and $-7 \mathrm{~V}$. The agreement with the experimental data is excellent. The desorption rate follows a powerlaw with exponent $\alpha \approx 6.5$. An analysis of the theoretical calculation shows that the contribution from $I_{2}$ dominates the desorption rate, and therefore $\alpha \approx N / 2$, where $N=13$ is the number of levels in the truncated harmonic potential well. This contrasts with the low positive bias case, 


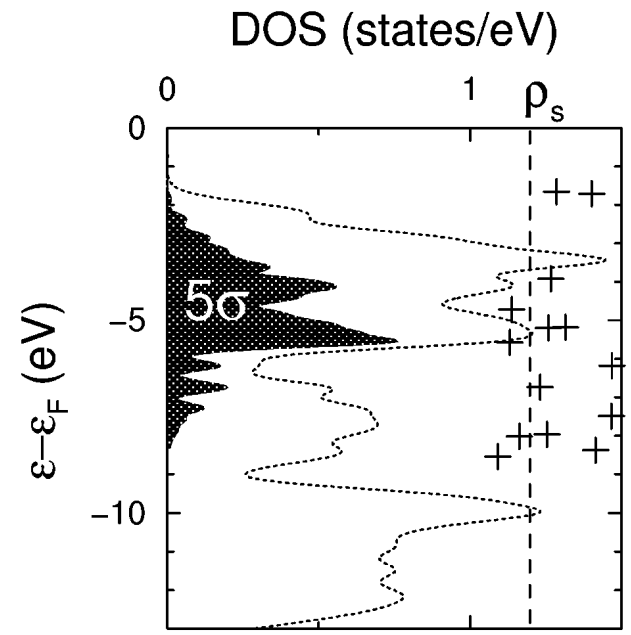

FIG. 4. The partial DOS, $n_{5 \sigma}(\varepsilon)=\sum_{\mu}|\langle 5 \sigma \mid \mu\rangle|^{2} \delta\left(\varepsilon-\varepsilon_{\mu}\right)$, of the $5 \sigma$ wave function of a $\mathrm{Si}-\mathrm{H}$ molecule. The dotted line shows the projection onto all $\mathrm{Si}-\mathrm{H}$ molecular wave functions. Crosses show values of $n_{5 \sigma}\left(\varepsilon_{\mu}\right) /\left.\langle 5 \sigma \mid \mu\rangle\right|^{2}$.

$V_{b}<4 \mathrm{~V}$, where $I_{1}$ dominates and $\alpha \approx 13$ [3]. This difference is due to the different lifetimes of the $5 \sigma$ and $6 \sigma^{*}$ resonances. Since the $6 \sigma^{*}$ resonance is more short-lived $(\Delta=1 \mathrm{eV})$, the energy transfer in each inelastic scattering event is smaller and $I_{1}$ dominates in this case [23]. Furthermore, electrons with energy $<4 \mathrm{eV}$ only sample the low energy tail of the $\mathrm{Si}-\mathrm{H} 6 \sigma^{*}$ resonance, and $\rho_{1}$ is relatively broader than $\rho_{n}$ for $n>1$; see Fig. 1 .

We next calculate $I_{\mathrm{des}}\left(V_{b}\right)$ and the results are shown in Fig. 3, and we note that the minimum at $-7 \mathrm{~V}$ is accurately reproduced by the theoretical calculation. This minimum does not coincide with the resonance energy $\varepsilon_{5 \sigma}$, but with the onset of the field emission regime, as illustrated by $I-V$ characteristics for this system at a constant field of $0.8 \mathrm{~V} / \AA$ (dashed line). In field $0.8 \mathrm{~V} / \AA$ surface states are band bent by $\sim 1 \mathrm{eV}$ [15] and therefore $\varepsilon_{5 \sigma} \approx 6 \mathrm{eV}$. Thus, in the range $[-6.5 \mathrm{~V}, 0 \mathrm{~V}]$ the bias dependence of $I_{\mathrm{des}}$ is mainly determined by the shape of the resonance wave function. Below $-6.5 \mathrm{~V}$ the bias dependence is a result of competition between two different effects: polarization of the hole resonance and increasing Fermilevel conduction. The electric field polarizes the surface, displacing wave functions towards the tip. Since the $5 \sigma$ orbital is more polarized than bulk states, its overlap with the tip state increases relative to other states, and the fraction of electrons causing double vibrational excitations, $f_{2}=I_{2} / I$, increases with electric field, as observed in the voltage range $[-7 \mathrm{~V},-6.5 \mathrm{~V}]$. On the other hand, the growing tip-sample distance with increasing magnitude of the negative bias will increase the tunnel barrier of the $5 \sigma$ state relative to states near the Fermi level, thus $f_{2}$ decreases with tip-sample distance. This effect becomes dominant at the onset of the field emission regime, where the conduction of states near the Fermi level is independent of the tip-sample distance, and $f_{2}$ starts to decrease when $V_{b}<-7 \mathrm{~V}$.

In conclusion, we have presented experimental measurements of the voltage and current dependent variation of the hydrogen desorption rate from the $\mathrm{Si}(100)-\mathrm{H}(2 \times$ 1) surface at negative bias conditions. Based on a novel first principles theory of inelastic scattering, we have shown that the desorption is caused by vibrational heating of the $\mathrm{H}$ atom due to inelastic-scattering with the $\mathrm{Si}-\mathrm{H}$ $5 \sigma$ hole resonance.

This work was supported by the Japanese Science and Technology Agency and the Danish Ministries of Industry and Research in the framework of the Center for Nanostructures (CNAST). The use of national computer resources was supported by the Danish Research Councils.

[1] J. A. Stroscio and D. M. Eigler, Science 254, 1319 (1991).

[2] P. Avouris, Acc. Chem. Res. 28, 95 (1995).

[3] T.-C. Shen et al., Science 268, 1590 (1995).

[4] B. C. Stipe et al., Phys. Rev. Lett. 78, 4410 (1997).

[5] G. P. Salam, M. Persson, and R. E. Palmer, Phys. Rev. B 49, 10655 (1994).

[6] D. P. Adams, T. M. Mayer, and B.S. Swartzentruber, J. Vac. Sci. Technol. B 14, 1642 (1996).

[7] J. W. Lyding et al., Appl. Phys. Lett. 64, 2010 (1994).

[8] S. Gao, M. Persson, and B. I. Lundqvist, Solid State Commun. 84, 271 (1992).

[9] J. G. Simmons, J. Appl. Phys. 34, 1793 (1963).

[10] R. H. Fowler and L. Nordheim, Proc. R. Soc. London A 119, 173 (1928).

[11] F. Perez-Murano et al. (unpublished).

[12] R. E. Walkup, D. M. Newns, and P. Avouris, in Atomic and Nanometer Scale Modification of Materials, edited by P. Avouris (Kluwer, Dordrecht, 1993).

[13] S. Gao, M. Persson, and B. I. Lundqvist, Phys. Rev. B 55, 4825 (1997).

[14] J. Tersoff and D. R. Hamann, Phys. Rev. B 31, 805 (1985).

[15] K. Stokbro, U. Quaade, and F. Grey, Report No. condmat/9710076 (1997).

[16] CRC Handbook of Chemistry and Physics, 75th Edition, edited by D. R. Lide (CRC Press, New York, 1994).

[17] P. Hohenberg and W. Kohn, Phys. Rev. 136, B864 (1964).

[18] W. Kohn and L. J. Sham, Phys. Rev. 140, A1133 (1965).

[19] J. P. Perdew et al., Phys. Rev. B 46, 6671 (1992).

[20] D. Vanderbilt, Phys. Rev. B 41, 7892 (1990).

[21] B. N. J. Persson, Phys. Scr. 38, 282 (1988).

[22] S. Gao, Phys. Rev. B 55, 1876 (1997).

[23] K. Stokbro et al., Report No. cond-mat/9802301 (1998).

[24] $I_{n}$ diverge for large $n$ [5], and the divergence depends on $\lambda / \Delta$. In the present calculation we use $n_{\max }=3$, but obtain quite similar results using $n_{\max }=2,3$ or 4 .

[25] P. Guyot-Sionnest, P. H. Lin, and E. M. Miller, J. Chem. Phys. 102, 4269 (1995). 